\title{
RELAÇÃO ENTRE CARACTERÍSTICAS MORFOLÓGICAS DE FRUTOS E INCIDÊNCIA DE PODRIDÃO CARPELAR EM CLONES DE MACIEIRA 'GALA' E 'FUJI' SOBRE DIFERENTES PORTA-ENXERTOS'
}

\author{
FABIANE NUNES SILVEIRA ${ }^{2}$, AIKE ANNELIESE KRETZSCHMAR ${ }^{3}$, \\ LEO RUFATO ${ }^{4}$, AMAURI BOGO $^{5}$, JOÃO CAETANO FIORAVANÇO ${ }^{6}$
}

RESUMO - A podridão carpelar tem-se tornado uma importante doença no Brasil, deixando de ser considerada uma doença secundária dentro do grupo das doenças de verão. O desenvolvimento e o formato dos frutos podem ser influenciados por eventos climáticos que ocorrem durante o período de polinização e frutificação da macieira. Além de outros fatores como nutrição das plantas, manejo da condução, tipo de porta-enxerto e cultivar copa. As alterações no formato dos frutos advindas destes fatores podem influenciar no aumento da intensidade da doença. O objetivo deste trabalho foi relacionar características morfológicas de frutos com a incidência de podridão carpelar, em clones de macieira, utilizando diferentes porta-enxertos, durante os ciclos de produção de 2009/2010 e 2010/2011, no município de Vacaria, no Estado do Rio Grande do Sul. O delineamento experimental foi em blocos casualizados, em arranjo fatorial 9 × 2, com nove clones das cultivares Gala e Fuji e dois porta-enxertos EM-9 e Marubakaido com interenxerto de EM-9. As características morfológicas dos frutos avaliados foram: relação entre comprimento e diâmetro de frutos $(\mathrm{C} / \mathrm{D})$, distância entre lóbulos, abertura calicinar, comprimento do tubo calicinar, número de sementes e classe de sintomas da doença nos carpelos. A doença não foi identificada nos clones da cultivar Gala, em ambos os ciclos de produção. Houve interação entre os fatores clones e porta-enxertos para as características morfológicas avaliadas, em ambos os ciclos de produção. A maior incidência da doença nos clones de 'Fuji' apresentou uma relação positiva com as características morfológicas dos frutos com maior abertura calicinar, menor relação C/D e maior distância entre lóbulos dos frutos, quando comparados aos clones de 'Gala'. O clone 'Fuji Suprema', enxertado sobre porta-enxerto EM-9, apresentou 19,33 \% de incidência de podridão carpelar, sendo significativamente superior quando comparado aos $6,67 \%$ de incidência do porta-enxerto Marubakaido com interenxerto de EM-9. Dentre os gêneros de fungos isolados de carpelos de maçãs, Alternaria é o gênero que apresentou maior frequência na proporção de 14 e 20\% nos ciclos de 2009/2010 e 2010/2011, respectivamente.

Termos de indexação: Malus domestica, abertura calicinar, Alternaria sp., distância entre lóbulos.

\section{RELATIONSHIP BETWEEN FRUIT MORPHOLOGICAL CHARACTERISTICS AND INCIDENCE OF MOLDY CORE IN 'GALA' AND 'FUJI'APPLE CLONES ON DIFFERENT ROOTSTOCKS}

\begin{abstract}
The moldy core has become an important disease in Brazil, no longer considered a secondary disease within the group of summer diseases. The development and fruit shape can be influenced by weather events that occur during the period of pollination and fruit set of apple trees. In addition to other factors such as plant nutrition, management of drives, type of rootstock and scion. The changes in fruits shape these factors can influence the increased intensity of disease. The objective of this study was to relate fruit morphological characteristics with the incidence of moldy core disease in different clones of apple Gala and Fuji cultivars on different rootstocks during the crop season of 2009/10 and 2010/11 in the municipality of Vacaria in the state of Rio Grande do Sul. The experimental design was completely randomized in a factorial 9 x 2 with nine clones of Gala and Fuji and two EM-9 rootstocks and interstock Marubakaido with EM-9. The fruit morphological characteristics evaluated were: relation between fruit length and diameter (L/D), distance between lobes, opening calicinar, calicinar tube length, seed number and class of disease symptoms in carpels. The disease was not identified in any clones of 'Gala' cultivars in both crop season. There was interaction between clones and rootstocks for all fruit morphological characteristics in both crop season 2009/10 and 2010/11. The highest incidence of disease in clones of 'Fuji' showed a positive relationship with the morphological characteristics of fruits with bigger calicinar openness, lower relationship between L/D and greater distance between lobes of the fruit, when compared with clones of 'Gala'. The clone 'Fuji Suprema' grafted on rootstock EM-9 presented 19,33\% of moldy core incidence that was significantly higher than $6,67 \%$ incidence for rootstock Marubakaido interstock with EM-9. Among the genera of fungi isolated from carpels of apples, Alternaria was the genus with the highest frequency in the proportion of 14 and 20\% in 2009/10 and 2010/11 crop season, respectively.
\end{abstract}

Index terms: Malus Domestica, opening calycine, Alternaria sp., distance between lobes.

\footnotetext{
1(Trabalho 185-12). Recebido em: 22-03-12. Aceito para publicação em:

${ }^{2}$ Eng. Agrônoma, Mestranda em Produção Vegetal, CAV/UDESC. Lages - SC. E-mail: fabinhans29@yahoo.com.br

${ }^{3}$ Dra. Fruticultura, Orientadora, Professora do Departamento de Agronomia, CAV/UDESC. Lages - SC. E-mail: a2aak@cav.udesc.br

${ }^{4}$ Dr. Fruticultura, Co-orientador, Professor do Departamento de Agronomia, CAV/UDESC. Lages-SC. Bolsista de Produtividade em Pesquisa do CNPq. E-mail: leoruffato@yahoo.com.br

${ }^{5}$ Dr. Fitopatologia, Professor do Departamento de Agronomia, CAV/UDESC. Lages - SC. E-mail: amauribogo@udesc.br

${ }^{6}$ Dr. Pesquisador EMBRAPA Uva e Vinho. Bento Gonçalves -RS. E-mail: fioravanço@cnpuv.embrapa.br
} 


\section{INTRODUÇÃO}

A área plantada com macieiras (Malus domestica Borkh.) no Brasil é de 39 mil ha (IBGE, 2011), tornando o Brasil destaque na cultura devido ao crescente aumento da área cultivada. As cultivares Gala e Fuji são as mais utilizadas, responsáveis por $90 \%$ da produção de maçã da região Sul do Brasil. Estes dois grupos de cultivares possuem clones, mutações surgidas espontaneamente, com características mais promissoras que a cultivar original. Essas mutações estão geralmente relacionadas a alterações da coloração da epiderme do fruto, embora também existam mutações relacionadas ao tamanho do fruto, vigor da planta, hábito de frutificação, resistência a doenças e época de produção (FIORAVANÇO et al., 2010).

A cultura da macieira atualmente dispõe de um grande número de porta-enxertos e dentre eles, os dois mais utilizados são o Marubakaido e o EM-9. Estes porta-enxertos possuem comportamento oposto em relação ao vigor conferido às plantas, sendo o primeiro bastante vigoroso, adaptado a diferentes tipos de solo, enquanto o segundo é ananizante, reduzindo o vigor da planta, o que facilita os tratos culturais, as aplicações de produtos fitossanitários e a colheita, conferindo ainda precocidade de frutificação, boa produtividade e boa qualidade dos frutos (DENARDI, 2006). O tipo de porta-enxerto utilizado pode ter influência nas características conferidas aos frutos, em relação a características de diâmetro e comprimento médio do fruto, número de sementes, entre outras (MATHIAS et al., 2008). Essas características possuem importância do ponto de vista fitopatológico, pois alterações no formato dos frutos podem predispor à maior incidência de podridão carpelar (KRETZSCHMAR et al., 2007).

A podridão carpelar é uma doença caracterizada pela formação de micélios de coloração preta ou cinza, dependendo do agente causal, ocupando todos os espaços vazios, sobre as sementes e paredes dos carpelos, na região das lojas carpelares. Essa doença pode ser causada por uma série de fungos, dos quais os mais frequentemente isolados são Alternaria alternata (Fr.) Keissler e Cladosporium herbarum (Pers.) Link.. A infecção ocorre através de partes da flor, e a doença pode desenvolver-se ainda no pomar, principalmente em cultivares com cálice aberto, como as do grupo 'Red Delicious' (SHENDEREY et al., 2010). Entretanto, poucos são os estudos comparando diferentes clones das cultivares Gala e Fuji sobre diferentes portaenxertos, relacionando o efeito das características morfológicas de frutos com a incidência de podridão carpelar. O objetivo deste trabalho foi relacionar características morfológicas de frutos com a incidência de podridão carpelar em diferentes clones das cultivares Gala e Fuji, sobre os porta-enxertos EM-9 e Marubakaido com interenxerto de EM-9 nas condições edafoclimáticas do município de VacariaRS, durante as safras 2009/2010 e 2010/2011.

\section{MATERIAL E MÉTODOS}

Os experimentos foram conduzidos durante os ciclos de produção de 2009/2010 e 2010/2011, em pomar conduzido no sistema de Produção Integrada de Maçã (PIM), da Estação Experimental de Fruticultura Temperada (EEFT) - Unidade da Embrapa Uva e Vinho, localizada na cidade de Vacaria-RS (Latitude $28^{\circ} 33^{\prime} \mathrm{S}$ e Longitude $50^{\circ}$ $\left.57^{\prime} \mathrm{W}\right)$, com altitude de 955 metros acima do nível do mar. O clima da região caracteriza-se como mesotérmico úmido $(\mathrm{Cfb})$, segundo a classificação de Köppen, com chuvas bem distribuídas durante todo o ano, temperaturas máximas médias de $22,4{ }^{\circ} \mathrm{C}$ e mínimas de $12,35^{\circ} \mathrm{C}$. O pomar foi implantado em 2006, os espaçamentos de plantio dos diferentes clones foram de 1,0 e 1,4 m entre plantas e 3,5 e 4,0 m entre linhas para o porta-enxerto EM-9 e Marubakaido, interenxertado com EM-9 (Maruba/EM-9), respectivamente. As plantas foram conduzidas sob sistema líder central em espaldeira. Os dados meteorológicos foram coletados na Estação Experimental de Vacaria - EMBRAPA Uva e Vinho.

Os clones da cultivar Gala avaliados foram 'Gala Real', 'Baigent', 'Maxi Gala', 'Royal Gala', 'Galaxy' e 'Imperial Gala', e da cultivar Fuji, 'Mishima', 'Fuji Select' e 'Fuji Suprema', e os portaenxertos EM-9 e o Maruba/EM-9. Foram avaliados 300 frutos de cada clone sobre seus respectivos porta-enxertos, sendo 150 avaliados na colheita e 150 avaliados 10 dias após a colheita. Os frutos dos clones da cultivar Gala foram colhidos na primeira quinzena do mês de fevereiro, e dos clones da cultivar Fuji,na primeira quinzena do mês de abril, em ambos os ciclos de produção de 2009/2010 e 2010/2011. Os frutos foram armazenados em caixas plásticas e mantidos em temperatura ambiente $\left(23-25^{\circ} \mathrm{C}\right)$ por 10 dias.

Os frutos foram avaliados quanto à relação comprimento/diâmetro (C/D) e, em seguida, cortados no sentido longitudinal e avaliados quanto à abertura calicinar, distância entre lóbulos, comprimento do tubo calicinar e número de sementes. Todas as medidas foram realizadas com auxílio de paquímetro digital. Para a avaliação da incidência de podridão carpelar, frutos com sintomas da doença foram 
classificados com auxílio de uma escala de sintomas elaborada por Kretzschmar (2004), sendo: infecção de sementes (IS), para aqueles frutos com micélio crescendo apenas sobre as sementes; infecção avançada (IA), para aqueles frutos com micélio ocupando toda a cavidade carpelar e podridão (P), para aqueles frutos com início de podridão na polpa até o ponto de exteriorização da podridão para a epiderme do fruto.

Fragmentos do tecido infectado da região carpelar foram depositados em placas de Petri com meio Batata-Dextrose-Ágar (BDA) mais estreptomicina $(0,01 \mathrm{~g} / \mathrm{L})$. As placas foram mantidas em câmara de germinação tipo BOD (Biochemical Oxygen Demand) a $26^{\circ} \mathrm{C}$, sob fotoperíodo de 16 horas, de 3 a 15 dias. A identificação dos patógenos foi realizada pela conidiogênese e morfologia dos esporos (BARNET; HUNTER, 1972).

O delineamento experimental foi de blocos ao acaso, em arranjo fatorial $9 \times 2$, com nove clones das cultivares Gala e Fuji e dois porta-enxertos, três repetições e dez plantas por unidade experimental.

Os dados obtidos foram submetidos à análise da variância (ANOVA) e, quando significativos, as médias foram analisadas pelo teste de Tukey, a 5\% de probabilidade de erro, através do programa estatístico WinStat 2.0.

\section{RESULTADOS E DISCUSSÃO}

Houve interação entre os clones de maçãs das cultivares Gala e Fuji e os porta-enxertos para as características morfológicas dos frutos, durante os ciclos de produção de 2009/2010 e 2010/2011 (Tabelas 1 e 2). A podridão carpelar não foi identificada em clones da cultivar Gala, independentemente dos porta-enxertos e dos ciclos de produção avaliados, sugerindo que estes clones são resistentes (ABE et al., 2010) à podridão carpelar (Tabela 1). Esta resistência pode estar relacionada às características morfológicas dos frutos, pois condições varietais, climáticas ou fisiológicas que podem alterar o formato dos frutos de macieira, podem afetar a incidência de podridão carpelar (PETRI, 2006; KRETZSCHMAR et al., 2007). Outros fatores como: menor conteúdo de compostos fenólicos e baixa atividade polifenoloxidase dos frutos (LI et al., 2011), teor de açúcar (TOURNAS; KATSOUDAS, 2005), época de maturação e precocidade de colheita dos frutos da cultivar Gala (AMARANTE et al., 2003) podem também estar relacionados com a não incidência da doença nos vários clones da cultivar Gala avaliados.

Com relação às variações observadas na incidência de podridão capelar, ns clones da cultivar
Fuji os dados nao foram conclusivos. No ciclo de produção de 2009/2010, não diferiram significativamente entre si e entre os diferentes porta-enxertos, apresentando apenas menor incidência no clone 'Fuji Suprema' quando enxertado sobre Maruba/EM-9. No ciclo de produção de 2010/2011, não houve diferença significativa na incidência entre os porta-enxertos, mas houve diferença na incidência entre os clones sobre porta-enxerto EM-9, e esta incidência foi de $17,33 \%, 8,00 \%$ e $28,67 \%$ para os clones 'Mishima', 'Fuji Select' e 'Fuji Suprema' sobre EM-9, e 19,33\%, $14,00 \%$ e $20,00 \%$ sobre Maruba/EM-9, respectivamente (Tabela 1). O clone 'Fuji Select' apresentou $8,00 \%$ de incidência sobre o porta-enxerto EM-9, significativamente menor quando comparado ao clone 'Fuji Suprema'. Não houve diferença significativa em relação à incidência da doença nos frutos avaliados logo após a colheita, em comparação com a incidência nos frutos avaliados no período de 10 dias após a colheita, em ambos os ciclos de produção.

A interação entre a cultivar copa e o porta-enxerto pode ter influência expressiva no crescimento vegetativo, na nutrição mineral da copa e na qualidade dos frutos (TOMALA et al., 2008). Características geneticamente determinadas pelos porta-enxertos podem ter efeito na firmeza dos frutos, sólidos solúveis e acidez titulável (SKRZYNSKI; GASTOL, 2007), eficiência no aporte de água e nutrientes (WOLPERT et al., 2005).

Entretanto, com relação a este efeito dos porta-enxertos e sua interação com os clones de macieira sobre a incidência da doença, pode-se considerar apenas como um possível fator indicativo de que, além da maior suscetibilidade dos clones de 'Fuji' à doença, a interação entre os clones e os porta-enxertos utilizados sobre as características morfológicas dos frutos também possa estar relacionada com a menor incidência da doença observada no clone 'Fuji Suprema' sobre o porta-enxerto Maruba/ EM-9, uma vez que esta diferença foi significativa apenas no ciclo de produção de 2009/2010, não se repetindo durante o segundo ciclo de produção em estudo. Os porta-enxertos interferem na fisiologia da planta, na adaptação a fatores ambientais, apresentando diferentes respostas de acordo com a copa em que eles são enxertados, sendo que este efeito pode variar ao longo dos ciclos de produção (DENARDI et al., 2006; DI VAIO et al., 2009).

Com relação às características morfológicas dos frutos, os clones 'Mishima', 'Fuji Select' e 'Fuji Suprema' apresentaram os maiores valores para a abertura calicinar dos frutos sobre ambos os porta-enxertos e em ambos os ciclos de produção, com exceção apenas para o clone 'Mishima' sobre o 
porta-enxerto Maruba/EM-9, durante o segundo ciclo de produção. No ciclo de produção de 2010/2011, os clones 'Fuji Suprema' e 'Mishima' sobre o porta-enxerto EM-9 apresentaram os maiores valores de abertura calicinar, assim como as maiores porcentagens de incidência da doença, em comparação ao clone 'Fuji Select', o qual, dentre os clones de 'Fuji', apresentou a menor incidência de podridão carpelar sobre o porta-enxerto EM-9. Houve correlação significativa $(\mathrm{p}<0,05)$ entre incidência de podridão carpelar e abertura calicinar dos frutos de clones de 'Fuji' apresentando coeficiente de correlação igual a 0,49. Archer (2002), em estudos avaliando a incidência de podridão carpelar e sua relação com a abertura calicinar, verificou que frutos de 'Red Delicious' e 'Red Fuji' apresentaram maior abertura calicinar e maior incidência da doença nos frutos. 'Golden Delicious', 'Pink Lady', 'Granny Smith' e 'Braeburn' apresentaram baixa incidência de podridão carpelar e menores valores para a abertura calicinar. Conforme Reuveni e Prusky (2007), a presença de maior abertura calicinar, associada à suscetibilidade da cultivar e à ocorrência de condições de temperaturas moderadas $\left(10\right.$ a $\left.25^{\circ} \mathrm{C}\right)$ com umidade relativa do ar alta (acima de 70\%) são condições ideais que favorecem a incidência da doença.

Durante o ciclo de produção de 2010/2011, no período que compreende a floração da macieira, a temperatura média foi de $15^{\circ} \mathrm{C}$, dois graus abaixo do observado no ciclo de 2009/2010, cuja temperatura média foi de $17^{\circ} \mathrm{C}$. A umidade relativa foi semelhante em ambos os ciclos de produção, atingindo valores médios de $80 \%$ e $76 \%$ durante os meses de agosto a novembro, nos ciclos de produção, de 2009/2010 e 2010/2011, respectivamente. O número de dias com precipitação no ciclo de 2009/2010 durante os meses de janeiro a abril, foi menor (11 dias) em comparação ao ciclo de 2010/2011 (14 dias). No período de maturação dos frutos, a precipitação média durante os meses de janeiro a abril, foi mais elevada no ciclo de 2010/2011 do que em 2009/2010, atingindo 212 $\mathrm{mm}$ e $165 \mathrm{~mm}$, respectivamente, o que pode ter contribuído para a maior porcentagem da doença neste ciclo de 2010/2011.

Para a relação $C / D$ dos frutos, os clones 'Mishima' e 'Fuji Select' apresentaram os menores valores para esta relação C/D sobre ambos os porta-enxertos, no ciclo de produção de 2009/2010. No ciclo de produção de 2010/2011, os clones 'Mishima', 'Fuji Select' e 'Fuji Suprema' apresentaram menores valores para a relação $\mathrm{C} / \mathrm{D}$ dos frutos e maior incidência de podridão carpelar nos frutos, diferindo dos demais clones de 'Gala', os que apresentaram maiores valores para a relação C/D dos frutos e au- sência de sintomas da doença nos frutos. A correlação entre a incidência de podridão carpelar nos clones de 'Fuji' e a relação C/D dos frutos foi significativa $(\mathrm{p}<0,05)$ somente no segundo ciclo de produção, apresentando valor de $\mathrm{r}$ igual a 0,58. Kretzschmar et al. (2007) relatam que a doença é mais comum em frutos com menor relação comprimento/diâmetro.

Com relação ao comprimento do tubo calicinar (Tabela 2) no ciclo de produção de 2009/2010, o clone 'Mishima' apresentou o menor valor para o comprimento do tubo calicinar em ambos os porta-enxertos. No ciclo de produção de 2010/2011, apenas com o porta-enxerto Maruba/EM-9 o clone 'Mishima' obteve novamente o menor valor para o comprimento do tubo calicinar. No entanto, com o uso do porta-enxerto EM-9, 'Fuji Suprema' apresentou o menor valor para esta variável, diferindo apenas de 'Baigent'. Dentre os clones de 'Fuji', houve correlação significativa $(\mathrm{p}<0,01)$ apenas no segundo ciclo de produção, cujo valor do coeficiente de correlação foi igual a -0,75. Esta correlação negativa entre a incidência da doença e o comprimento do tubo calicinar sugere que, quanto menor o comprimento do tubo calicinar do fruto, maior a relação com a ocorrência de podridão carpelar. Este resultado corrobora com os resultados encontrados por Gajardo et al. (1994), em seu estudo, onde se avaliaram frutos de macieira das cultivares Red King Oregon, Starkrimson e Richard, e observaram que existe relação entre a incidência de podridão carpelar e o comprimento e largura do tubo calicinar, assim como a relação comprimento/ largura do tubo calicinar.

Para a distância entre lóbulos dos frutos nos clones 'Mishima', 'Fuji Select' e 'Fuji Suprema', observou-se maior distância entre os lóbulos do fruto em comparação aos clones da cultivar Gala, em ambos os ciclos de produção e em ambos os porta-enxertos utilizados (Tabela 2), indicando que a maior distância entre lóbulos do fruto está associada com a maior incidência da doença nos clones de 'Fuji', em comparação à não incidência nos clones de 'Gala', que apresentaram menores valores para a distância entre lóbulos do fruto, em ambos os porta-enxertos e em ambos os ciclos de produção avaliados. Houve correlação significativa $(\mathrm{p}<0,05)$ entre a incidência da doença e a distância entre lóbulos do fruto, nos clones de 'Fuji', apresentando coeficiente de correlação igual a 0,47 . Embora no segundo ciclo de produção esta correlação não tenha sido significativa, estes resultados indicam haver correlação positiva entre esta característica morfológica e a incidência da doença, porque frutos dos clones de 'Fuji' com maior distância entre lóbulos apresentaram maior porcentagem de incidência da podridão carpelar. Isto 
concorda com resultados obtidos por Kretzschmar et al. (2007), que observaram que a podridão carpelar é mais frequente em frutos com maior distância entre lóbulos. Testando a influência de diferentes fitorreguladores na incidência de podridão carpelar, a autora observou que a aplicação de thiadizuron, utilizado por pomicultores para estimular o crescimento e o formato das frutas, aumentou a distância entre lóbulos dos frutos.

Em relação ao número de sementes por fruto, o clone 'Mishima' apresentou os menores valores quando enxertado em EM-9, assim como um reduzido número de sementes quando enxertado em Maruba/EM-9, não diferindo estatisticamente dos menores valores obtidos por 'Fuji Select' em 2009/2010 e 'Galaxy' em 2010/2011. A correlação entre a incidência da doença e a variável número de sementes por fruto não foi significativa em ambos os ciclos de produção avaliados. Entretanto, segundo Boneti e Katsurayama (1999), a maior incidência de podridão carpelar foi observada em frutos de macieira que apresentaram problemas relacionados à polinização, e com isso a formação das sementes pode ser reduzida, não garantindo boa formação dos frutos, devido à redução na síntese de hormônios que são produzidos pelas sementes.

Com relação à classificação dos sintomas da doença, a maior incidência durante o ciclo de produção de 2009/2010 ocorreu com o sintoma caracterizado pela fase inicial da podridão carpelar, quando se observa apenas infecção de sementes (IS), com micélios presentes na região dos lóculos das sementes. Observou-se também, em menor incidência, o sintoma classificado como infecção avançada (IA), com presença de micélio ocupando toda a cavidade carpelar dos frutos. Não foi observada a terceira classe de podridão (P) (Figura 1A). Durante o ciclo, de produção de 2010/2011, a maior incidência da doença foi novamente caracterizada pelo sintoma em que a doença se manifesta na forma de infecção de sementes (IS). Durante este ciclo ocorreu a terceira classe denominada podridão (P), na qual se observa um início de podridão na polpa até o surgimento de exteriorização desta podridão para a epiderme do fruto (Figura 1B). Isto concorda com os resultados obtidos por Kretzschmar (2004), que avaliou a distribuição das porcentagens de sintomas da doença em maçãs 'Fuji', oriundas de cinco pomares diferentes na região de Vacaria-RS, e verificou que a maior incidência da doença foi obtida na fase inicial da doença, na região dos lóculos das sementes.

Com relação à frequência dos gêneros de fungos isolados de carpelos de maçãs 'Fuji', na região de Vacaria-RS, durante o ciclo de 2009/2010, verificou-se que a maior frequência foi do gênero Alternaria sp., seguida de Fusarium sp., Ulocladium sp., Geotrichum sp., Phoma sp. e, em menor frequência, Colletotrichum sp. e Pestalotia sp. (Figura 2C). Segundo Kretzschmar (2004), foram identificados carpelos de maçãs 'Fuji', oriundas de cinco pomares da região de Vacaria-RS, dois deles sobre Produção Integrada e três sobre Produção Convencional, os seguintes gêneros de fungos: Alternaria sp. em maior frequência, Fusarium sp., Gliocladium sp., Phoma sp., Colletotrichum sp., Pestalotia sp. e Cladosporium sp. Reuveni et al. (2007) citam que os sintomas de podridão carpelar em maçãs 'Star King Delicious', causados por Alternaria, podem estar relacionados à maior capacidade de Alternaria produzir endo-1,4-b-glucanase (EG) durante o processo de infecção nos frutos.

Durante o ciclo de 2010/2011, a maior frequência foi novamente do gênero Alternaria sp. (Figura 2D), seguida de Phoma sp., Fusarium sp., Criptosporium sp e Colletotrichum sp e, em menor frequência Gliocladium sp., Geotrichum sp. e $M a-$ crophoma sp. Atualmente, em isolamentos realizados na região de Israel, na Ásia, sintomas de podridão carpelar em frutos de maçã têm sido comumente associados com Pleospora herbarum sp. e espécies de Stemphylium sp., Cladosporium sp., Ulocladium sp., Epicoccum sp., Coniothyrium sp., Penicillium sp., Pezicula sp., Fusarium sp., Mucor sp., e Alternaria sp. (NIEM et al., 2007). 
TABELA 1 - Incidência de podridão carpelar, abertura calicinar e relação comprimento/diâmetro de frutos de diferentes clones de 'Gala' e 'Fuji' sobre dois porta-enxertos, avaliados 10 dias após a colheita em Vacaria, durante os ciclos de produção de 2009/2010 e 2010/2011. Lages, 2011.

\begin{tabular}{|c|c|c|c|c|}
\hline \multirow[b]{3}{*}{ Clones } & \multicolumn{4}{|c|}{ Incidência (\%) } \\
\hline & \multicolumn{2}{|c|}{$2009 / 2010$} & \multicolumn{2}{|c|}{$2010 / 2011$} \\
\hline & EM-9 & Maruba/EM-9 & EM-9 & Maruba/EM-9 \\
\hline Gala Real & 0 & 0 & 0 & 0 \\
\hline Baigent & 0 & 0 & 0 & 0 \\
\hline Maxi Gala & 0 & 0 & 0 & 0 \\
\hline Royal Gala & 0 & 0 & 0 & 0 \\
\hline Galaxy & 0 & 0 & 0 & 0 \\
\hline Imperial Gala & 0 & 0 & 0 & 0 \\
\hline Mishima & 16,00 a $\mathrm{A}$ & 8,67 a $A$ & $17,33 \mathrm{ab} \mathrm{A}$ & 19,33 a A \\
\hline Fuji Select & 9,33 a A & 8,00 a $\mathrm{A}$ & $8,00 \mathrm{~b} \mathrm{~A}$ & 14,00 a A \\
\hline Fuji Suprema & 19,33 a A & 6,67 a B & 28,67 a A & 20,00 a A \\
\hline Média & 14,88 & 7,78 & 21,78 & 17,78 \\
\hline \multirow[t]{2}{*}{ CV(\%) } & 56,72 & 56.72 & 46,71 & 46,71 \\
\hline & \multicolumn{4}{|c|}{ Abertura calicinar (mm) } \\
\hline Gala Real & $0,41 \operatorname{cd~A}$ & $0,24 \mathrm{~b} \mathrm{~A}$ & $1,32 \mathrm{~d} \mathrm{~B}$ & $1,87 \mathrm{bc} \mathrm{A}$ \\
\hline Baigent & $0,01 \mathrm{~d} A$ & $0,17 \mathrm{~b} \mathrm{~A}$ & $1,50 \mathrm{~cd} A$ & 1,49 с A \\
\hline Maxi Gala & $0,95 \mathrm{~cd} \mathrm{~A}$ & 0,02 b B & $1,35 \mathrm{~d} \mathrm{~A}$ & $1,39 \mathrm{c} \mathrm{A}$ \\
\hline Royal Gala & $0,28 \mathrm{~cd} \mathrm{~A}$ & $0,13 \mathrm{~b} \mathrm{~A}$ & $1,58 \mathrm{~cd} \mathrm{~A}$ & $1,82 \mathrm{bc} \mathrm{A}$ \\
\hline Galaxy & $0,08 \mathrm{~d} \mathrm{~A}$ & $0,05 \mathrm{~b} \mathrm{~A}$ & $1,48 \mathrm{~cd} \mathrm{~A}$ & $1,61 \mathrm{bc} \mathrm{A}$ \\
\hline Imperial Gala & $0,13 \mathrm{~d} A$ & $0,04 \mathrm{~b} \mathrm{~A}$ & $1,40 \mathrm{~d} A$ & $1,40 \mathrm{c} \mathrm{A}$ \\
\hline Mishima & $1,36 \mathrm{bc} B$ & 2,14 a A & $2,22 \mathrm{ab} A$ & 1,86 bc B \\
\hline Fuji Select & $2,11 \mathrm{~b} \mathrm{~A}$ & 1,82 a A & $1,97 \mathrm{bc} \mathrm{A}$ & 2,88 a A \\
\hline Fuji Suprema & 3,41 a A & 1,42 a B & 2,64 a A & $2,12 \mathrm{~b} \mathrm{~A}$ \\
\hline Média & 0,97 & 0,67 & 1,71 & 1,82 \\
\hline CV $(\%)$ & 49,04 & 49,04 & 10,77 & 10,77 \\
\hline
\end{tabular}

\begin{tabular}{lcccc}
\hline \multicolumn{5}{c}{ Relação comprimento/diâmetro do fruto (C/D) } \\
\hline Gala Real & $0,883 \mathrm{~cd} \mathrm{~A}$ & $0,892 \mathrm{abc} \mathrm{A}$ & $0,904 \mathrm{ab} \mathrm{A}$ & $0,895 \mathrm{~b} \mathrm{~A}$ \\
Baigent & $0,923 \mathrm{ab} \mathrm{A}$ & $0,903 \mathrm{ab} \mathrm{B}$ & $0,932 \mathrm{a} \mathrm{A}$ & $0,875 \mathrm{~b} \mathrm{~B}$ \\
Maxi Gala & $0,906 \mathrm{bc} \mathrm{A}$ & $0,921 \mathrm{a} \mathrm{A}$ & $0,919 \mathrm{ab} \mathrm{A}$ & $0,900 \mathrm{ab} \mathrm{A}$ \\
Royal Gala & $0,893 \mathrm{bcd} \mathrm{A}$ & $0,905 \mathrm{ab} \mathrm{A}$ & $0,867 \mathrm{c} \mathrm{B}$ & $0,890 \mathrm{~b} \mathrm{~A}$ \\
Galaxy & $0,867 \mathrm{~d} \mathrm{~A}$ & $0,865 \mathrm{~cd} \mathrm{~A}$ & $0,899 \mathrm{bc} \mathrm{A}$ & $0,894 \mathrm{~b} \mathrm{~A}$ \\
Imperial Gala & $0,948 \mathrm{a} \mathrm{A}$ & $0,915 \mathrm{a} \mathrm{B}$ & $0,918 \mathrm{ab} \mathrm{A}$ & $0,930 \mathrm{a} \mathrm{A}$ \\
Mishima & $0,800 \mathrm{e} \mathrm{B}$ & $0,832 \mathrm{de} \mathrm{A}$ & $0,816 \mathrm{~d} \mathrm{~A}$ & $0,813 \mathrm{~cd} \mathrm{~A}$ \\
Fuji Select & $0,808 \mathrm{e} \mathrm{A}$ & $0,822 \mathrm{e} \mathrm{A}$ & $0,789 \mathrm{~d} \mathrm{~A}$ & $0,793 \mathrm{~d} \mathrm{~A}$ \\
Fuji Suprema & $0,875 \mathrm{~cd} \mathrm{~A}$ & $0,880 \mathrm{bc} \mathrm{A}$ & $0,815 \mathrm{~d} \mathrm{~A}$ & $0,828 \mathrm{c} \mathrm{A}$ \\
\hline Média & 0,878 & 0,881 & 0,873 & 0,868 \\
CV(\%) & 1,37 & 1,37 & 1,38 & 1,38
\end{tabular}

* Médias seguidas pela mesma letra minúscula nas colunas e maiúscula nas linhas, para cada ciclo de produção, não diferem entre si,pelo teste de Tukey $(\mathrm{p}>0,05)$. 
TABELA 2 - Comprimento do tubo calicinar, distância entre lóbulos e número de sementes por fruto de diferentes clones de 'Gala' e 'Fuji' sobre dois porta-enxertos, avaliados 10 dias após a colheita em Vacaria, durante os ciclos de produção de 2009/2010 e 2010/2011. Lages, 2011.

\begin{tabular}{|c|c|c|c|c|}
\hline \multirow[b]{3}{*}{ Clones } & \multicolumn{4}{|c|}{ Comprimento do tubo calicinar (mm) } \\
\hline & \multicolumn{2}{|c|}{$2009 / 2010$} & \multicolumn{2}{|c|}{$2010 / 2011$} \\
\hline & EM-9 & Maruba/EM-9 & EM-9 & Maruba/EM-9 \\
\hline Gala Real & $6,76 \mathrm{ab} \mathrm{A}$ & $7,01 \mathrm{ab} A$ & $5,34 \mathrm{ab} B$ & 8,17 a $\mathrm{A}$ \\
\hline Baigent & $7,84 \mathrm{ab} \mathrm{A}$ & $7,35 \mathrm{ab} A$ & 7,08 a $\mathrm{A}$ & 5,64 bc B \\
\hline Maxi Gala & $6,54 \mathrm{~b} \mathrm{~A}$ & $7,37 \mathrm{ab} A$ & $5,46 \mathrm{ab} A$ & 5,98 bc A \\
\hline Royal Gala & 8,43 a A & 8,05 a A & $6,61 \mathrm{ab} A$ & 6,34 abc $\mathrm{A}$ \\
\hline Galaxy & 7,19 ab A & $7,18 \mathrm{ab} \mathrm{A}$ & $6,34 \mathrm{ab} \mathrm{A}$ & $7,03 \mathrm{ab} \mathrm{A}$ \\
\hline Imperial Gala & 7,98 ab A & 7,75 a A & $5,49 \mathrm{ab} B$ & $7,05 \mathrm{ab} A$ \\
\hline Mishima & $4,46 \mathrm{c} \mathrm{A}$ & 4,72 с A & $5,44 \mathrm{ab} \mathrm{A}$ & $4,78 \mathrm{c} \mathrm{A}$ \\
\hline Fuji Select & $7,70 \mathrm{ab} \mathrm{A}$ & $5,73 \mathrm{bc} B$ & $6,46 \mathrm{ab} A$ & 5,13 bc B \\
\hline Fuji Suprema & $7,48 \mathrm{ab} \mathrm{A}$ & $6,69 \mathrm{ab} A$ & 4,62 b A & 5,14 bc A \\
\hline Média & 7,15 & 6,87 & 5,87 & 6,14 \\
\hline \multirow[t]{2}{*}{ C V (\%) } & 8,92 & 8,92 & 12,72 & 12,72 \\
\hline & \multicolumn{4}{|c|}{ Distância de lóbulos (mm) } \\
\hline Gala Real & $23,93 \mathrm{~b} \mathrm{~A}$ & 23,72 bcd A & $24,12 \mathrm{c} \mathrm{A}$ & 25,46 bcd A \\
\hline Baigent & $23,71 \mathrm{~b} \mathrm{~A}$ & $23,33 \mathrm{~cd} \mathrm{~A}$ & 24,49 bc A & $22,46 \mathrm{~d} A$ \\
\hline Maxi Gala & $22,29 \mathrm{~b} \mathrm{~A}$ & $23,23 \mathrm{~cd} \mathrm{~A}$ & 22,77 c A & $22,39 \mathrm{~d} A$ \\
\hline Royal Gala & $23,18 \mathrm{~b} \mathrm{~A}$ & $23,30 \mathrm{~cd} \mathrm{~A}$ & $24,51 \mathrm{bc} \mathrm{A}$ & $23,67 \mathrm{~cd} \mathrm{~A}$ \\
\hline Galaxy & $23,52 \mathrm{~b} \mathrm{~A}$ & $23,09 \mathrm{~d} A$ & $24,58 \mathrm{bc} \mathrm{A}$ & 26,47 c A \\
\hline Imperial Gala & $22,98 \mathrm{~b} \mathrm{~A}$ & $25,39 \mathrm{bcd} A$ & 25,16 abc A & $24,02 \mathrm{~cd} \mathrm{~A}$ \\
\hline Mishima & 34,07 a A & 31,32 a B & 28,55 a A & $27,17 \mathrm{bc} \mathrm{A}$ \\
\hline Fuji Select & 30,31 a $\mathrm{A}$ & $27,38 \mathrm{abc} B$ & $27,83 \mathrm{ab} \mathrm{A}$ & $27,91 \mathrm{ab} A$ \\
\hline Fuji Suprema & 33,55 a A & $27,73 \mathrm{ab} \mathrm{B}$ & 28,09 ab B & 31,00 a A \\
\hline Média & 26,39 & 25,38 & 25,56 & 25,61 \\
\hline \multirow[t]{2}{*}{ CV(\%) } & 6,02 & 6,02 & 5,35 & 5,35 \\
\hline & \multicolumn{4}{|c|}{ Número de sementes } \\
\hline Gala Real & $6,48 \mathrm{ab} A$ & 6,50 a $\mathrm{A}$ & $5,87 \mathrm{ab} \mathrm{A}$ & 5,78 abc $\mathrm{A}$ \\
\hline Baigent & 7,25 a A & $5,52 \mathrm{ab} B$ & $6,24 \mathrm{ab} A$ & $5,90 \mathrm{ab} A$ \\
\hline Maxi Gala & $5,98 \mathrm{ab} A$ & 6,80 a $\mathrm{A}$ & $6,35 \mathrm{ab} A$ & 6,54 a A \\
\hline Royal Gala & $6,30 \mathrm{ab} A$ & 6,53 a $A$ & $6,22 \mathrm{ab} A$ & 6,06 a $A$ \\
\hline Galaxy & $6,72 \mathrm{ab} A$ & 6,84 a A & $5,89 \mathrm{ab} A$ & 4,75 c B \\
\hline Imperial Gala & $6,21 \mathrm{ab} A$ & 6,80 a $A$ & $6,58 \mathrm{ab} A$ & 6,02 a $A$ \\
\hline Mishima & 4,42 c B & $5,67 \mathrm{ab} A$ & $5,64 \mathrm{~b} \mathrm{~A}$ & 5,44 abc $\mathrm{A}$ \\
\hline Fuji Select & $5,32 \mathrm{bc} \mathrm{A}$ & 4,76 b A & $6,15 \mathrm{ab} A$ & 5,58 abc A \\
\hline Fuji Suprema & $6,01 \mathrm{ab} \mathrm{A}$ & 6,52 a A & 6,87 a $\mathrm{A}$ & 4,82 bc B \\
\hline Média & 6,07 & 6,21 & 6,20 & 5,65 \\
\hline CV (\%) & 9,05 & 9,05 & 7,12 & 7,12 \\
\hline
\end{tabular}

* Médias seguidas pela mesma letra minúscula nas colunas e maiúscula nas linhas, para cada ciclo de produção, não diferem entre si, pelo teste de Tukey $(\mathrm{p}>0,05)$. 


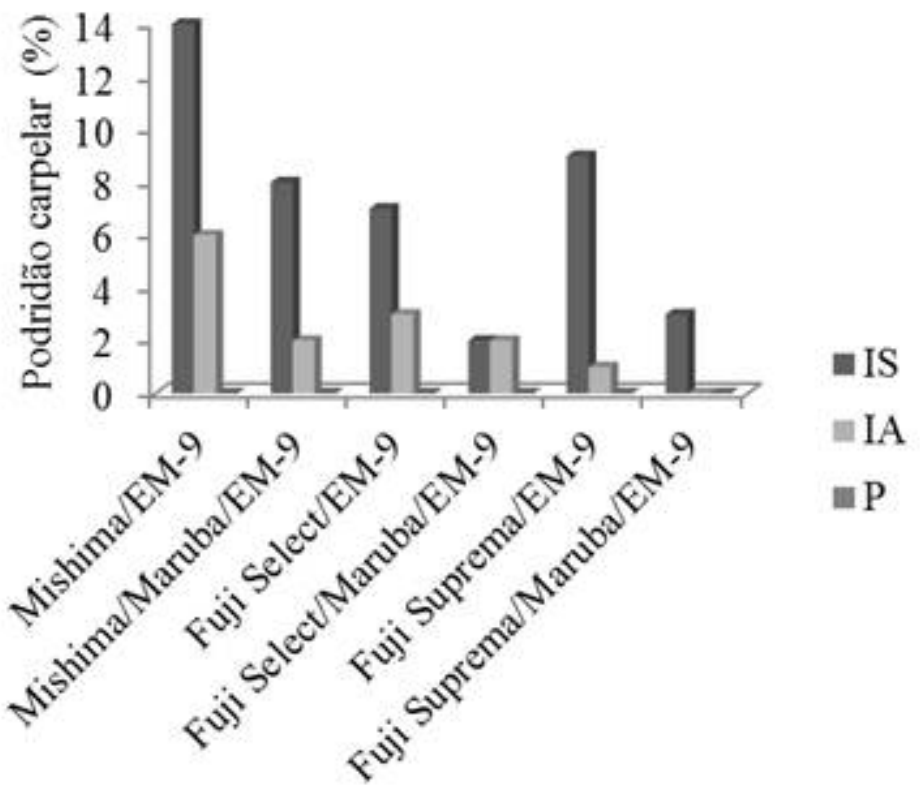

A

Clones/portaenxertos

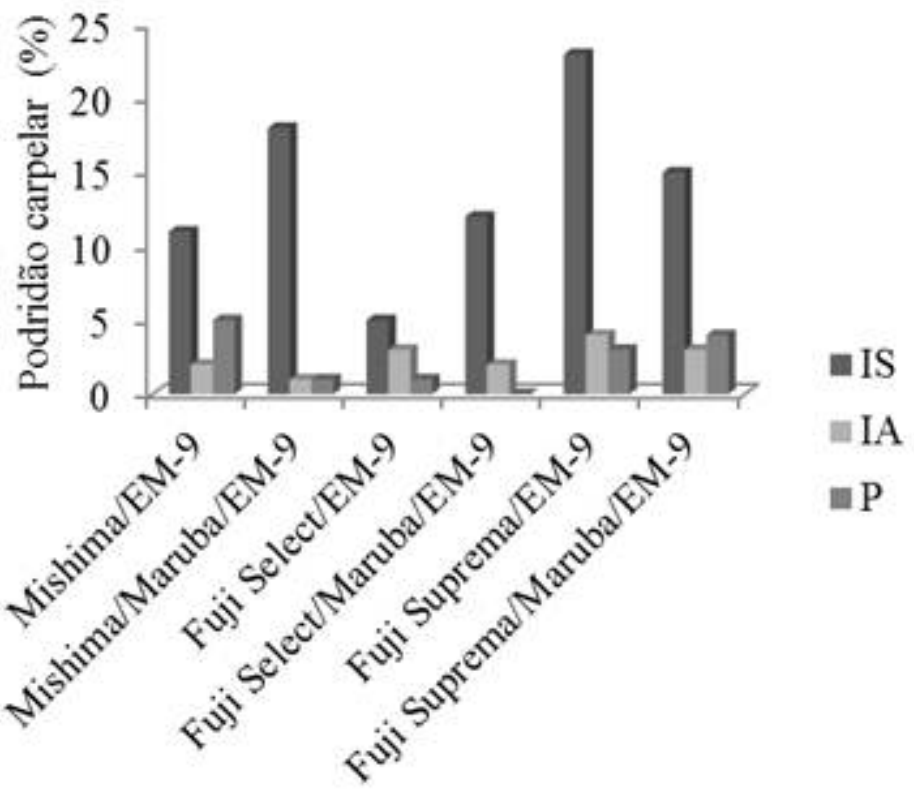

B

\section{Clones/portaenxertos}

FIGURA 1 - Porcentagem de frutos com sintomas da doença classificados de acordo com cada classe em: infecção de sementes (IS), infecção avançada (IA) e podridão (P), observados em carpelos de maçãs avaliadas 10 dias após a colheita, durante a safra de 2009/2010 (A) e durante a safra de 2010/2011 (B). Lages, 2011. 

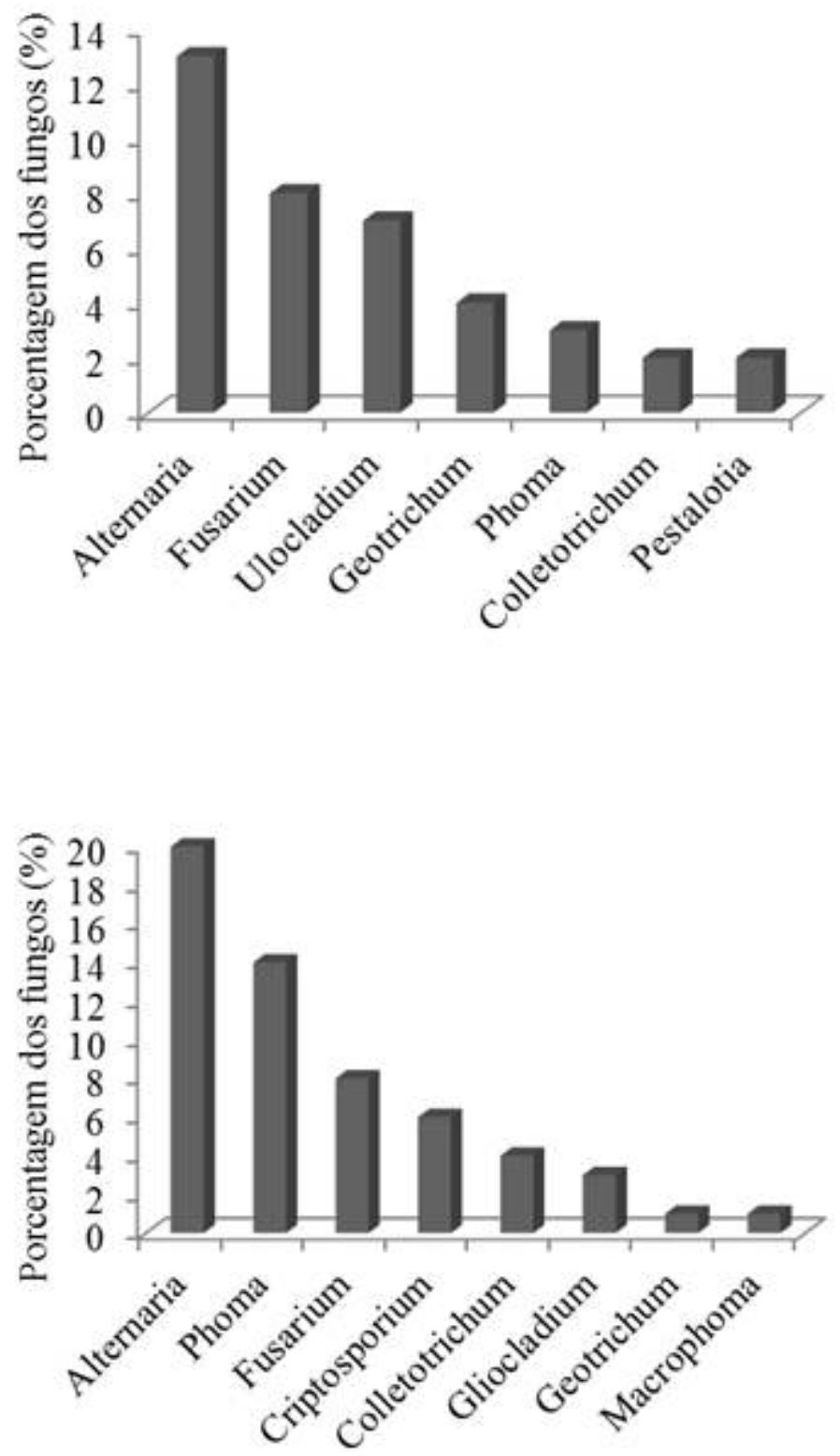

FIGURA 2 - Porcentagem dos gêneros de fungos isolados a partir dos carpelos de maçãs dos clones de 'Fuji', na região de Vacaria-RS, durante a safra de 2009/2010 (C) e durante a safra de 2010/2011 (D). Lages, 2011. 


\section{CONCLUSÕES}

1-A podridão carpelar não foi identificada em frutos de macieira de clones da cultivar Gala, independentemente do porta-enxerto e do ciclo de produção.

2-Frutos dos clones da cultivar Fuji com características morfológicas de maior abertura calicinar, menor relação C/D e maior distância entre lóbulos, em comparação aos clones da cultivar Gala, apresentam relação com a incidência da doença.

3-O gênero de fungo Alternaria é o que apresenta maior frequência em carpelos de frutos de macieira. O sintoma característico da doença é verificado principalmente nas sementes.

\section{REFERÊNCIAS}

ABE, K.; IWANAMI, H.; KOTODA, N.; MORIYA, S.; TAKAHASHI, S. Evaluation of apple genotypes and Malus species for resistance to Alternaria blotch caused by Alternaria alternata apple pathotype using detached-leaf method. Plant Breeding, Westport, v. 129, n. 2, p. 208-218, 2010.

AMARANTE, C.V.T.; MEGGUER, C. A; BLUM, L.E.B. Effect of preharvest spraying with thidiazuron on fruit quality and maturity of apples. Revista Brasileira de Fruticultura, Jaboticabal, v. 25, n. 1, p.59-62, 2003.

ARCHER, C. The use of honeybees a transfer vector for core rot in apples. 2002. 47f. A Report for the Rural Industries Research and Development Corporation. RIRDC project \#TAR-1A. RIRDC Publication.

BARNET, H. L.; HUNTER, B. B. Ilustrated genera of imperfect fungi. $3^{\text {rd }}$ ed. Minnesota: Burgess Publishing Company, 1972. 241 p.

BONETI, J. I.; KATSURAYAMA, Y. Podridão Carpelar da Macieira. In: REUNIÃO ANUAL DE FITOSSANIDADE NACULTURADAMACIEIRA, 1998/99, São Joaquim, SC. Relatório... São Joaquim: Epagri, Estação Experimental de São Joaquim, 1999. $40 \mathrm{p}$.

DENARDI, F. Porta-enxertos. In: EPAGRI. A cultura da macieira. Florianópolis, 1006. cap. 6, p. 169-227.
DI VAIO, C.; CIRILLO, C.; BUCCHERI, M.; LIMONGELLI, F. Effect of interstock (M.9 and M.27) on vegetative growth and yield of apple trees (cv "Annurca"). Scientia Horticulturae, Amsterdam, v. 119, n. 3, p. 270-274, 2009.

FIORAVANÇO, J. C.; GIRARDI, C. L.; CZERMAINSKI, A.B. C.; SILVA, G. A.; NACHTIGALL, G. R.; OLIVEIRA, P. R. D. Cultura da macieira no Rio Grande do Sul: análise situacional e descrição varietal. Bento Gonçalves: Embrapa Uva e Vinho, 2010. v. 2, p. 10-60. (Documentos, 71).

GAJARDO, C. E.; CANEO, M. L.; LUCCHINI, C. M. Incidencia de corazon mohoso del manzano en Talca, Linares y Chillan (Chile), y su relacion con las caracteristicas morfologicas del fruto e indices de

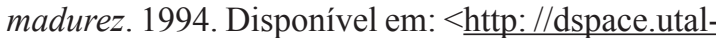
ca.cl/handle/1950/149>. Acesso em: 12 out. 2011.

IBGE - Instituto Brasileiro de Geografia e Estatística. Banco de dados agregados: orçamentos familiares. Disponível em: $<$ http://www.sidra.ibge.gov.br/bda/ orcfam/default.asp > . Acesso em: 15 abr. 2011.

KRETZSCHMAR, A. A. Fatores associados à podridão carpelar em maçã "Fuji" no Brasil. 2004. 133 f. Tese (Doutorado) - Faculdade de Agronomia, Universidade Federal do Rio Grande do Sul, Porto Alegre, 2004.

KRETZSCHMAR, A. A. MARODIN, G. A. B.; DUARTE, V.; VALDEBENITO-SANHUEZA, R. M.; GUERRA, D. S. Efeito de fitorreguladores sobre a incidência de podridão carpelar em maçãs 'Fuji'. Revista Brasileira de Fruticultura, Jaboticabal, v. 29, n. 3, p. 414-419, 2007

LI, S.; ZHANG, L.; LIU, X. Effects of mouldy core and core rot on physiological and biochemical responses of apple fruit. Journal of the Science of Food and Agriculture, London, v, 91, n. 14, p. 2674-2678, 2011

MATHIAS, C.; MAYER, N. A.; MATTIUZ, B. H.; PEREIRA, F. M. Efeito de porta-enxertos e espaçamentos entre plantas na qualidade de pêssegos 'Aurora. Revista Brasileira de Fruticultura, Jaboticabal, v. 30, n. 1, p. 165-170, 2008. 
NIEM, J., MIYARA, I., ETTEDGUI, Y., REUVENI, M., FLAISHMAN, M., and PRUSKY, D. Core rot development in red delicious apples is affected by susceptibility of the seed locule to Alternaria alternata colonization. Phytopathology, Saint Paul, v. 97, p. 1415-1427, 2007.

REUVENI, M. \& PRUSKY D. Improved control of moldy-core decay (Alternaria alternata) in Red Delicious apple fruit by mixtures of DMI fungicides and captan. European Journal of Plant Pathology, Dordrecht, v. 118, p. 349-357, 2007.

REUVENI, M.; SHEGLOV, N.; ESHEL, D.; PRUSKY D.; BEN-ARIE, R. Virulence and the production of Endo -1,4-B- glucanase by isolates Alternaria alternata involved in the moldy core disease of apples. Phytopathology, Saint Paul, v. 155, p. 50-55, 2007.

SHENDEREY, C.; SHMULEVICH, I.; ALCHANATIS, V.; EGOZI, H.; HOFFMAN, A.; OSTROVSKY, V.; LURIE, S.; BEN AIRE, R.; SCHMILOVITCH, $Z$. Nirs detection of moldy core in apples. Food Bioprocess Tecnologic, New York. v. 3, p. 79-86, 2010.
SKRZYNSKI, J.; GASTOL, M. The effect of rootstocks on the fruit characteristic attributes of 'Jonica' apples. Vegetable Crops Research Bulletin, Warsaw, v. 66, p. 171-176, 2007.

TOURNAS, V. H. \& KATSOUDAS, E. Mould and yeast flora in fresh berries, grapes and citrus fruits. International Journal of Food Microbiology, Amsterdam, v.105, n.1, p. 11-17, 2005.

TOMALA, K.; ANDZIAK, J.; JEZIOREK, K.; DZIUBAN, R. Influence of rootstocks on the quality of 'Jonagold' apples at harvest and after storage.

Journal of Fruit and Ornamental Plant Research, Skierniewice, v. 16, p. 31-38, 2008.

WOLPERT, J. A.; SMART, D. R.; ANDERSON, M. Lower petiole potassium concentration at bloom in rootstocks with Vitis berlandieri genetic backgrounds. American Journal of Enology and Viticulture, Davis, v. 56, p. 163-169. 2005. 